\title{
III. On the force on a magnetic particle in a magnetic field
}

\section{Prof. A. Anderson M.A. LL.D.}

To cite this article: Prof. A. Anderson M.A. LL.D. (1904) III. On the force on a magnetic particle in a magnetic field, Philosophical Magazine Series 6, 8:43, 38-42, DOI:

10.1080/14786440409463170

To link to this article: http://dx.doi.org/10.1080/14786440409463170

Published online: 15 Apr 2009.

Submit your article to this journal $₫$

Џll Article views: 6

Q⿳亠丷厂犬

View related articles $₫$ 


\section{$\left[\begin{array}{ll}38 & ]\end{array}\right.$}

III. On the Force on a Magnetic Particle in a Magnetic Field. By Prof. A. Anderson, M.A., LL.D.*

A SMALL particle of soft iron, or a small magnet, when
direction in which the intensity of the field increases most
rapidly. This is, in general, only true when the field is
conservative.
Consider a magnetic particle, $P$ (fig. 1), whose axis of Fig. 1.

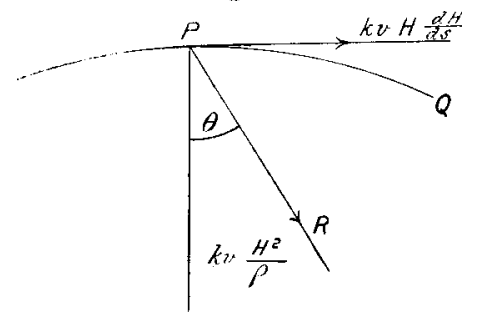

magnetization is along a line of force, PQ. It is easy to show that the forces acting along the tangent and normal are, respectively,

$$
\mathrm{M} \frac{d \mathrm{H}}{d s} \text { and } \mathrm{M} \frac{\mathrm{H}}{\rho},
$$

where $M$ is the moment of the particle, $H$ the intensity of the field, and $\rho$ the radius of curvature of the line of force at $P$.

If the particle be of small susceptibility, these expressions may be written

$$
k v \mathrm{H} \frac{d \mathrm{H}}{d s} \text { and } k v \frac{\mathrm{H}^{2}}{\rho},
$$

$v$ denoting the volume, and $k$ the susceptibility.

Let the resultant, $\mathrm{R}$, of these forces make an angle $\theta$ with the normal. Then, if this direction is that along which the intensity of the magnetic field increases most rapidly, the rate of increase perpendicular to $\mathrm{R}$ is zero, or

And hence

$$
\frac{d \mathrm{H}}{d s} \cos \theta-\frac{d \mathrm{H}}{d n} \sin \theta=0 .
$$

$$
\frac{\mathrm{H}}{\rho}=\frac{d \mathrm{H}}{d n} .
$$

* Communicated by the Author. 
Horce on a Magnetic Particle in a Magnetic Field.

Let $\mathrm{PQ}, \mathrm{P}^{\prime} \mathrm{Q}^{\prime}$ (fig. 2) be two indefinitely close lines of

Fig. ‥ -

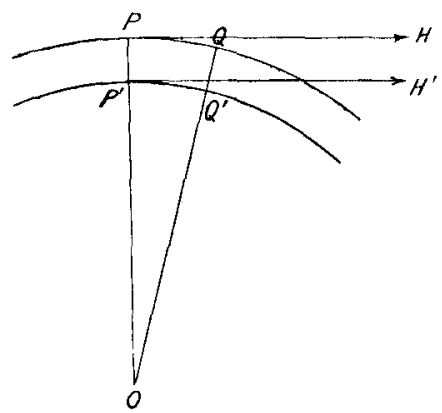

magnetic force, and let $\mathrm{H}$ be the magnetic intensity at $\mathrm{P}$ and $\mathrm{H}^{\prime}$ that at $\mathrm{P}^{\prime}, \mathrm{PP}^{\prime}$ and $\mathrm{QQ}^{\prime}$ being normals to the line of force at $P$ and $Q$. Then, since

it easily follows that

$$
\frac{\mathrm{H}}{\rho}=\frac{d \mathrm{H}}{d n},
$$

$$
\frac{\mathrm{H}}{\mathrm{P}^{\prime} \mathrm{Q}^{\prime}}=\frac{\mathrm{H}^{\prime}}{\overline{\mathrm{PQ}}} \text {. }
$$

The work done in carrying unit pole round the circuit $\mathrm{PQQ}^{\prime} \mathrm{P}^{\prime} \mathrm{P}$ is, therefore, zero; from which it follows that the field of force is conservative.

As an example of a non-conservative field, take the case of a current of electricity in a straight wire or cylindrical liquid column of circular cross section. The intensity of field inside, on the supposition that the current is uniformly distributed over the area of the cross section, is $\frac{2 \mathrm{Cr} r}{a^{2}}$, where $r$ is the distance from the axis and $a$ the radius of the wire. The force on a particle of soft iron inside is $\frac{4 k v \mathrm{C}^{2} r}{a^{4}}$, but it is in the direction in which the magnetic intensity diminishes most rapidly. Inside a wire conveying a current of electricity a magnetic potential does not exist, and the field is nonconservative.

We may now go a step farther and find the direction of the force on a magnetic particle in a plane field of given magnetic intensity, assuming that there exists a distribution of electric currents at right angles to the plane. Let the direction of the current be the positive direction through 
$\mathrm{PQQ}^{\prime} \mathrm{P}^{\prime}$. We have, easily,

$$
\frac{\mathrm{H}}{\rho}-\frac{d \mathrm{H}}{d n}=4 \pi w,
$$

where $w$ is the current density per unit area.

Let PX (fig. 3) making an angle $\phi$ with the normal PO

Fig. 3.

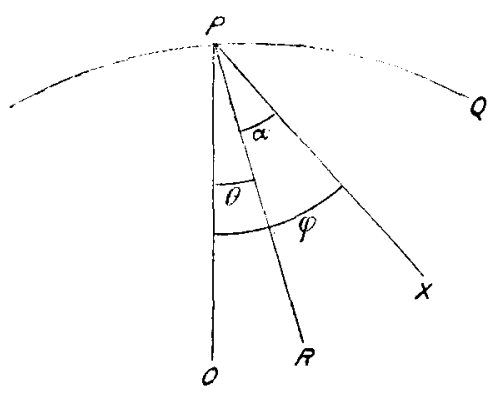

to the line of force at $\mathbf{P}$, be the direction in which the magnetic intensity increases most rapidly, and PR, making an angle $\theta$ with $\mathrm{PO}$, the direction of the force on a magnetic particle at $\mathrm{P}$. Also, let the angle $\mathrm{XPR}=\alpha$.

Then

$$
\tan \theta=\frac{d \mathrm{H}}{d s} / \frac{\mathrm{H}}{\rho},
$$

and

$$
\tan \phi=\frac{d \mathrm{H}}{d s} / \frac{d \mathrm{H}}{d n} ;
$$

from which it follows that

$$
\tan \alpha=\frac{4 \pi \rho w \sin \phi \cos \phi}{\bar{H}-4 \pi \frac{\pi}{\rho w \sin ^{2} \phi}} .
$$

If $\phi=0, \alpha=0$, which is the case for a particle outside a straight wire carrying a current.

If $\phi=\pi, \alpha=\pi$, which is the case for a particle inside a wire carrying a current.

To solve the general case, let $u, v, w$ be the components of current density at any point, $\alpha, \beta, \gamma$ the components of magnetic intensity, $\lambda, \mu, \nu$ the direction cosines of the direction in which the magnetic intensity increases most rapidly, and $\lambda^{\prime}, \mu^{\prime}, \nu^{\prime}$ the direction cosines of the force on the particle. 
Then

$\frac{\lambda}{\alpha \frac{d a}{d x}+\beta \frac{d \beta}{d x}+\gamma \frac{d \gamma}{d x}}=\frac{\mu}{\alpha \frac{d \alpha}{d y}+\beta \frac{d \beta}{d y}+\gamma \frac{d \gamma}{d y}}=\frac{\nu}{\alpha \frac{d \alpha}{d z}+\beta \frac{d \beta}{d z}+\gamma \frac{d \gamma}{d z}}$,

and

$\frac{\lambda^{\prime}}{\alpha \frac{d \alpha}{d x}+\beta \frac{d \alpha}{d y}+\gamma \frac{d a}{d z}}=\frac{\mu^{\prime}}{\alpha \frac{d \beta}{d x}+\beta \frac{d \beta}{d y}+\gamma \frac{d \beta}{d z}}=\frac{\nu^{\prime}}{\alpha \frac{d \gamma}{d x}+\beta \frac{d \gamma}{d y}+\gamma \frac{d \gamma}{d z}}$.

These equations, with the relations

$$
4 \pi u=\frac{d \gamma}{d y}-\frac{d \beta}{d z}, \quad 4 \pi v=\frac{d \alpha}{d z}-\frac{d \gamma}{d x}, \quad 4 \pi w=\frac{d \beta}{d x}-\frac{d \alpha}{d y},
$$

are sufficient to find the angle between the force on a magnetic particle and the direction in which the magnetic intensity increases most rapidly, the field being due to permanent magnets, and to a given distribution of currents.

It can easily be seen that the relation

$$
\left|\begin{array}{ccc}
\lambda, & \lambda^{\prime}, & \beta w-\gamma v \\
\mu, & \mu^{\prime}, & \gamma u-\alpha w \\
\nu, & \nu^{\prime}, & \alpha v-\beta u
\end{array}\right|=0
$$

holds between the components of magnetic intensity and current and the two directions considered. Hence if a line be drawn perpendicular to both the current and magnetic intensity, a magnetic particle will be urged in a direction lying in a plane through this line and the direction in which the magnetic intensity increases most rapidly.

Queen's College, Galway.

March 30, 1904.

\section{Postscript.}

The complete solution may be put as follows:-

Let $\lambda, \mu, \nu$ be the direction cosines of the direction of greatest increase of the magnetic intensity $H, \lambda^{\prime}, \mu^{\prime}, \nu^{\prime}$ those of the force $\mathrm{F}$ on the magnetic particle; then

$$
\begin{aligned}
p \lambda & =\frac{1}{2} \frac{d \mathrm{H}^{2}}{d x}, \quad p \mu=\frac{1}{2} \frac{d \mathrm{H}^{2}}{d y}, \quad p \nu=\frac{1}{2} \frac{d \mathrm{H}^{2}}{d z}, \\
p^{\prime} \lambda^{\prime} & =\frac{1}{2} \frac{d \mathrm{H}^{2}}{d x}+4 \pi(v \gamma-w \beta), \\
p^{\prime} \mu^{\prime} & =\frac{1}{2} \frac{d \mathrm{H}^{2}}{d y}+4 \pi(w \alpha-\imath \gamma), \\
v^{\prime} \nu^{\prime} & =\frac{1}{2} \frac{d \mathrm{H}^{2}}{d z}+4 \pi(u \beta-v \alpha) .
\end{aligned}
$$


Thus $p=\mathrm{H} \dot{\mathrm{H}}$, where $\dot{\mathrm{H}}$ is the maximum rate of increase of magnetic intensity.

Draw a line perpendicular to both the current density and magnetic intensity, its direction being that of translation of a right-handed screw rotated from current to magnetic intensity, and let its direction cosines be $\lambda^{\prime \prime}, \mu^{\prime \prime}, \nu^{\prime \prime}$. Then if $\mathrm{C}$ be the current density, and $\phi$ the angle between $\mathrm{C}$ and $\mathrm{H}$,

$$
\begin{aligned}
& p^{\prime} \lambda_{,}^{\prime}=p \lambda+4 \pi \mathrm{CH} \sin \phi \lambda^{\prime \prime}, \\
& p^{\prime} \mu^{\prime}=p \mu+4 \pi \mathrm{CH} \sin \phi \mu^{\prime \prime}, \\
& p^{\prime} \nu^{\prime}=p \nu+4 \pi \mathrm{CH} \sin \phi \nu^{\prime \prime} ;
\end{aligned}
$$

$\therefore p^{\prime 2}=p^{2}+8 \pi \mathrm{CH} p \sin \phi\left(\lambda \lambda^{\prime \prime}+\mu \mu^{\prime \prime}+\nu \nu^{\prime \prime}\right)+16 \pi^{2} \mathrm{C}^{2} \mathrm{H}^{2} \sin ^{2} \phi$; or if $\psi$ denote the angle between $(\lambda, \mu, \nu)$ and $\left(\lambda^{\prime \prime}, \mu^{\prime \prime}, \nu^{\prime \prime}\right)$,

$$
p^{\prime 2}=\mathrm{H}^{2}\left[\dot{\mathrm{H}}^{2}+8 \pi \mathrm{C} \sin \phi \cos \psi \dot{\mathrm{H}}+16 \pi^{2} \mathrm{C}^{2} \sin ^{2} \phi\right] .
$$

The force F, as has been shown above, lies in the plane through $(\lambda, \mu, \nu)$ and $\left(\lambda^{\prime \prime}, \mu^{\prime \prime}, \nu^{\prime \prime}\right)$. Let it make an angle $\psi^{\prime}$ with $\left(\lambda^{\prime \prime}, \mu^{\prime \prime}, \nu^{\prime \prime}\right)$; then it follows at once that

Thus

$$
p^{\prime} \cos \psi^{\prime}-p \cos \psi=4 \pi \mathrm{CH} \sin \phi \text {. }
$$

$$
\cos \psi^{\prime}=\frac{1 \dot{\mathrm{H}} \cos \psi+4 \pi \mathrm{C} \sin \phi}{\left[\dot{\mathrm{H}}^{2}+8 \pi \mathrm{C} \sin \phi \cos \psi \dot{\mathrm{H}}+16 \pi^{2} \mathrm{C}^{2} \sin ^{2} \phi\right]^{\frac{1}{2}}} .
$$

When $\mathrm{C}=0$, or $\phi=0, \psi^{\prime}=\psi$, the direction of $\mathrm{F}$ in both these cases being that in which the magnetic intensity increases most rapidly.

If $v=$ volume of the particle and $k$ the susceptibility supposed small, it is easily shown that

$$
\mathrm{F}=k v \mathrm{H}\left[\dot{\mathrm{H}}^{2}+8 \pi \mathrm{C} \sin \phi \cos \psi \dot{\mathrm{H}}+16 \pi^{2} \mathrm{C}^{2} \sin ^{2} \phi\right]^{\frac{1}{2}},
$$

which reduces to the well-known expression

$$
k v \mathrm{H} \dot{\mathrm{H}} \text {, when } \phi \text { or } \mathrm{C} \text { vanishes. }
$$

The expression for $\cos \psi^{\prime}$ leads to the very simple result that $\mathrm{F}$ is the resultant of two forces, one, equal to $k v \mathrm{H} \dot{\mathrm{H}}$, along the direction in which the magnetic intensity increases most rapidly, and the other, equal to $4 \pi k v \mathrm{HC} \sin \phi$, along the line perpendicular to the current and magnetic intensity. 